\title{
Anti-mitochondrial Therapy: A Potential Therapeutic Approach in Oncology
}

\author{
Farzad Taghizadeh-Hesary ${ }^{1,2}$, Hassan Akbari ${ }^{3,4}$, Moslem Bahadori 5 \\ 1. Department of Radiation Oncology, Iran University of Medical Sciences, Tehran, Iran. \\ 2. ENT and Head \& Neck Research Center and Department, The Five Senses Health Institute, Hazrat Rasoul Akram \\ Hospital, Iran University of Medical Sciences, Tehran, Iran \\ 3. Department of Pathology, Shahid Beheshti University of Medical Sciences, Tehran, Iran. \\ 4. Traditional Medicine School, Tehran University of Medical Sciences, Tehran, Iran. \\ 5. Professor emeritus, Faculty of Medicine, Tehran University of Medical Sciences, Tehran, Iran.
}

\section{Correspondence:}

1. Farzad Taghizadeh-Hesary, M.D.

Primary E-mail:f_taghizadeh@sbmu.ac.ir

Secondary E-mail: Farzadth89@gmail.com

Cell phone: +989126086713

Tel: +9826651517

Fax: +9826651517

Assistant Professor, Department of Oncology, Iran University of Medical Sciences, Tehran, Iran

ORCiD: 0000-0002-6195-2203

2. Hassan Akbari, M.D. PhD

E-mail address: hmhakbari@yahoo.com

Cell phone: +989121785345

Associate Professor, Department of Pathology, Shahid Beheshti University of Medical Sciences, Tehran, Iran.

Traditional Medicine School, Tehran University of Medical Sciences, Tehran, Iran. 


\title{
Anti-mitochondrial Therapy: A Potential Therapeutic Approach in Oncology
}

\begin{abstract}
Like living organisms, cancer cells require energy to survive and interact with their environment. Recently, investigators demonstrated that cancer cells can hijack mitochondria from immune cells. This behavior sheds light on a pivotal piece in the puzzle of cancer, the 'dependence'. This article illustrates how new, functional mitochondria help cancer cells' survival in the harsh tumor microenvironment, immune evasion, and progression. In addition, we postulate how blocking the routes cancer cell applies to boost its mitochondria content and function can improve the treatment outcomes of radiotherapy, chemotherapy, and immunotherapy. Besides, this article provides a new theory in oncology, the 'energy battle' between cancer and immune cells. It alludes that each party with a higher energy level can win the battle. This theory explains cancer biogenesis and provides novel insights to improve treatment outcomes.
\end{abstract}

Keywords: ATP; Cancer cell; Cancer Treatment; Mitochondria; T cell

\section{Introduction}

All living organisms require energy for their maintenance, growth, repopulation, and appropriate response to external stimuli. Some organisms are self-sufficient ('autotrophs') and acquire energy from sunlight or chemicals. The remaining organisms ('heterotrophs') rely on autotrophs to secure energy ${ }^{1}$. A recent in vitro experiment from the United States demonstrated that cancer cells are dependent on normal cells for their living and function. In Nov 2021, Saha et al. demonstrated that cancer cells can hijack mitochondria (the cell's energy factories) from immune cells via nanoscale tube-like structures ${ }^{2}$. Besides providing energy, mitochondria are essential organelles for cancer cells' survival and evolution.

This study aims to provide a comprehensive overview of mitochondria's pivotal role in cancer metabolism. Throughout this paper, we demonstrate how mitochondria are involved in cancer cells' survival in the tumor microenvironment, immune evasion, malignancy, and treatment resistance. The following section explains the mitochondria's multifaceted role in cancer metabolism and describes how functional mitochondria are vital for cancer survival and progression. 


\section{Mitochondria's Benefits for Cancer Cells}

Mitochondria bring four crucial benefits for cancer cells (Figure 1);

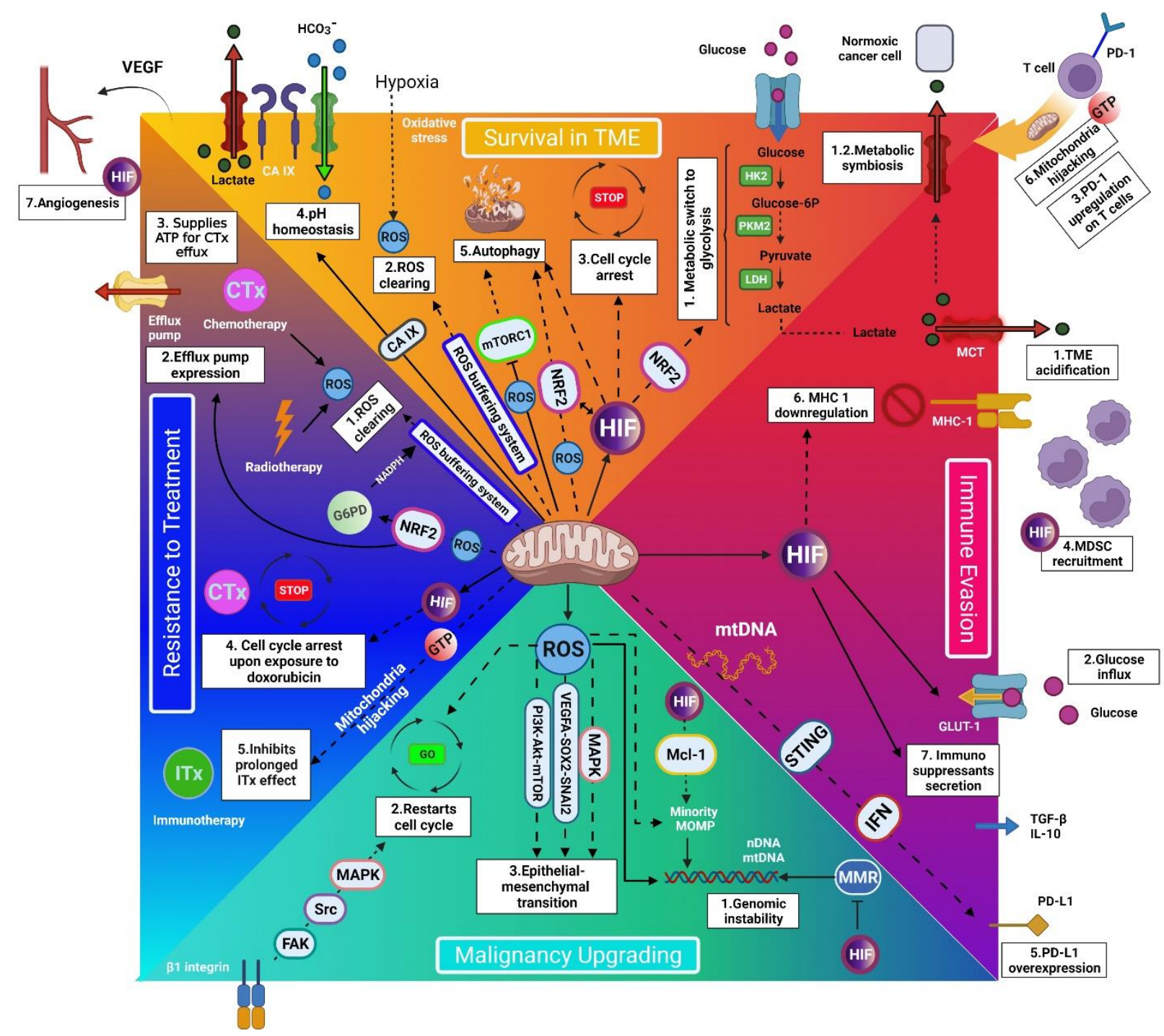

Figure 1. Schematic model of mitochondria role in cancer survival, immune evasion, progression, and treatment resistance. The white boxes depict the mitochondria regulation outcomes. (A) Survival in the tumor microenvironment (TME) (orange area): functional mitochondria are requisite for cancer cells to survive in the harsh TME by facilitating/mediating (A1) glycolysis, (A2) ROS clearing, (A3) cell cycle arrest, (A4) enhanced pH homeostasis, (A5) autophagy, (A6) mitochondrial hijacking, and (A7) angiogenesis. (B) Immune evasion (pink area): mitochondria assist cancer cells in evading the immune cells by mediating (B1) TME acidification, (B2) glucose influx, (B3) PD-1 upregulation on T cells (by mitochondrial hijacking), (B4) recruiting myeloid-derived suppressor cells (MDSCs), (B5) PD-L1 expression on cancer cells, (B6) MHC-1 downregulation, and (B7) immunosuppressant secretion. Besides, mitochondrial hijacking from $\mathrm{T}$ cells depletes T cells' energy and impedes long-term activity against cancer. (3) Malignancy upgrading (light blue area): mitochondria are essential for cancer progression by mediating (C1) genomic instability, (C2) quiescence evasion, and (C3) epithelial-to-mesenchymal transition. These actions are mediated by reactive oxygen species (ROS) production. (D) Resistance to treatment (dark blue area): (D1) mitochondria can serve 
as a defense shield for cancer cells against radiotherapy and chemotherapy by clearing ROS. (D2-4) Besides, they improve chemotherapy resistance by mediating efflux pump expression, providing ATP for efflux pumps, and inducing cell cycle arrest. (D5) in addition, mitochondria hijacking from $\mathrm{T}$ cells impairs the long-term effect of anti-PD-1 immunotherapy.

Note: The HIF- and GTP-mediated extracellular outcomes are shown in their corresponding white boxes. Abbreviations: ATP, adenosine triphosphate; CA IX, carbonic anhydrase IX; EMT, epithelial-mesenchymal transition; FAK/Src/MAPK, focal adhesion kinase/Src/mitogen-activated protein kinase; GLUT-1, Glucose transporter-1; GTP, guanosine triphosphate; G6PD, glucose 6-phosphate dehydrogenase; HIF, hypoxiainducible factor; HK2, hexokinase 2; IFN, interferon; IL-10, interleukin-10; LDH, lactate dehydrogenase; MDSC, myeloid-derived suppressor cell; MHC-1, major histocompatibility complex class I; mTORC1, mechanistic target of rapamycin complex 1; mtDNA, mitochondrial DNA; NADPH, nicotinamide adenine dinucleotide phosphate; NRF2, nuclear factor-erythroid 2 related factor 2; PI3K/Akt/mTOR, phosphatidylinositol-3kinase/protein kinase B/mammalian target of rapamycin; PD-1, programmed cell death protein-1; PD-L1, programmed cell death protein-ligand 1; PKM2, pyruvate kinase M2; ROS, reactive oxygen species; STING, stimulator of interferon genes; TGF- $\beta$, transforming growth factor-beta; TME, tumor microenvironment; VEGF, vascular endothelial growth factor; VEGFA/SOX2/SNAI2, vascular endothelial growth factor A-SRY-Box Transcription Factor 2.

\subsection{Surviving in the Harsh Tumor Microenvironment}

Hypoxia threatens human cells by hampering mitochondrial ATP production and excessive reactive oxygen species (ROS) accumulation ${ }^{3}$. Cancer cells can cope with a hypoxic tumor microenvironment (TME) by (1) metabolic switch to glycolysis, (2) enhanced redox homeostasis, (3) protective cell cycle arrest, (4) pH homeostasis, (5) autophagy, (6) mitochondria hijacking, and (7) promoting angiogenesis 2,4,5. Accumulating evidence indicates that mitochondria are involved in the strategies mentioned above. This section summarizes the current understanding of the role of mitochondria in tumor hypoxia resistance.

2.1.1. Metabolic switch to glycolysis: Cancer cells preserve the ATP/ADP ratio in hypoxia by a metabolic switch from oxidative phosphorylation (OXPHOS) to anaerobic glycolysis. This phenomenon persists in normoxia, which is known as aerobic glycolysis ${ }^{6}$. Hypoxiainducible factor- $1 \alpha$ (HIF- $1 \alpha$ ) is the master regulator of adaptation to hypoxia. In hypoxia, HIF-1 $\alpha$ improves the expression of glycolytic enzymes, including hexokinase 2 (HK2) (the rate-limiting enzyme of glycolysis) and pyruvate kinase M2 (PKM2). In breast cancer cells, HIF-1 $\alpha$ promotes glycolysis by upregulating nuclear factor-erythroid 2 related factor 2 (NRF2) ${ }^{7}$. In addition, HIF-1 $\alpha$ prevents pyruvate from entering the tricarboxylic acid (TCA) cycle. This action is mediated by activating pyruvate dehydrogenase kinase 1 (PDK1), which in turn impedes pyruvate conversion to acetyl-CoA (the substrate of the TCA cycle) by inhibiting pyruvate dehydrogenase (PDH) ${ }^{8}$. Functional mitochondria enable cancer cells to increase glycolytic flux by stabilizing HIF- $1 \alpha$ and facilitating its function ${ }^{9}$. The sustained glycolytic pathway provides three benefits for cancer cells: (1) aerobic glycolysis can satisfy the anabolic demands of cancer cells by providing lipids, proteins, and nucleotides ${ }^{10} ;(2)$ the pyruvates (interim products of aerobic glycolysis) can serve as an antioxidant and neutralizes the intracellular ROS - as a byproduct of cellular metabolism ${ }^{11}$; and (3) 
normoxic cancer cells can utilize lactate (final products of glycolysis) as an energy source (known as 'metabolic symbiosis') ${ }^{10}$.

2.1.2. Redox homeostasis: ROS accumulates in normal cells under hypoxia conditions. An in vitro study on hepatocellular carcinoma cells demonstrated that cancer cells could cope with this condition by removing the accumulated ROS ${ }^{6}$. Mitochondria are involved in enhanced redox homeostasis of cancer cells in the following ways: (1) Li et al. demonstrated that mitochondria are involved in this process by upregulating antioxidant enzymes (e.g., glutathione reductase, glutathione peroxidase, and glutaredoxins) and redox buffering systems (e.g., glutathione) ${ }^{6}$; (2) glutathione buffering system requires NADPH to remain reduced. The primary source of NADPH is the pentose phosphate pathway, in which glucose 6-phosphate dehydrogenase (G6PD) is the rate-limiting enzyme ${ }^{6}$. G6PD is directly activated by NRF2, which is upregulated by mitochondrial ROS (mtROS) - denoting mitochondria metabolism 7,12; besides, (3) mitochondria can assist to neutralize the ROS by HIF-dependent glycolytic flux to produce more pyruvates ${ }^{11}$. Experimental evidence demonstrates the antioxidant capacity of pyruvate ${ }^{13-15}$.

2.1.3. Protective cell cycle arrest (dormancy or quiescence): it is an ability that cancer cells adapt to survive in harsh TME, contributing to tumor recurrence. Dormancy is characterized by mitotic arrest at G0/G1 phase ${ }^{16}$. A study on colon cancer cells indicated that dormancy is through HIF-dependent p21 and p27 overexpression (two CDK-cyclin inhibitors) ${ }^{17}$. Recent evidence has shown the mitochondria's reaction to the hypoxic condition. In an in vitro model of dormant breast cancer cells, chronic hypoxia led to a marked increase in mitochondria content and biogenesis ${ }^{18}$. This finding put forward the notion that mitochondria are involved in the regulatory machinery of tumor dormancy.

2.1.4. $\mathrm{pH}$ homeostasis: Besides hypoxia, acidic $\mathrm{pH}$ is another characteristic of TME. This condition is intolerable for normal cells and leads them to apoptosis. However, cancer cells can tolerate acidic $\mathrm{pH}$ by employing a transmembrane glycoprotein called carbonic anhydrase IX (CA IX). It contributes to cancer cells to preserve physiologic $\mathrm{pH}$ through bicarbonate influx in cooperation with sodium bicarbonate cotransporters (NBC) and lactate efflux in cooperation with monocarboxylate transporters (MCT). CA IX is expressed in a wide array of cancer types, including glioblastoma, breast, colorectal, lung, and cervical cancer ${ }^{19}$. A study on osteosarcoma cells revealed that mitochondria directly regulate CA IX function ${ }^{9}$.

2.1.5. Autophagy: In the stressful hypoxic TME, cancer cells preserve cellular homeostasis by degrading and recycling cytoplasmic proteins, lipids, and nonfunctional organelles. A large body of evidence noted that functional mitochondria promote cancer cells to autophagy by increasing intracellular ROS level, which inactivates the mechanistic target of rapamycin complex 1 (mTORC1) (an autophagy inhibitor) on the one hand, and activates NRF2 (an autophagy activator) on the other hand ${ }^{20-22}$. Given the following two wellestablished assumptions, one might put forward another mechanism by which 
mitochondria are involved in autophagy: (1) hypoxia-inducing autophagy is mediated by HIF- $1 \alpha^{23}$, (2) mitochondria stabilizes HIF- $1 \alpha$ and facilitates its function ${ }^{9}$. In breast cancer cells, NRF2 knockdown leads to HIF dysregulation in mediating autophagia ${ }^{7}$. This finding indicates a cross-talk between NRF2 and HIF-1 $\alpha$ in regulating autophagy in cancer cells.

2.1.6. Mitochondria hijacking: A recent study on Lewis lung carcinoma cells revealed that cancer cells generate nanoscale tubes to hijack the T cells' mitochondria. ${ }^{2}$. This capability enables cancer cells to replace the old, defective mitochondria (degraded by mitophagy) with the new, functional mitochondria from immune cells to reply to the mitochondria demands. The existing mitochondria of cancer cells can potentially mediate mitochondria hijacking from normal cells by considering the following assumptions: (1) Upon tunneling nanotube formation, the interaction between mitochondrial Rho GTPase (Miro1) and actins - inside the nanotubes-mediates mitochondria migration from normal cells toward cancer cells. This process is GTP-dependent ${ }^{2}$. (2) It has been indicated that mitochondria's TCA cycle is the main source of cellular GTP ${ }^{24}$.

2.1.7. Angiogenesis: In a restrictive TME, cancer cell implicates strategies to find access to oxygen and nutrients supporting its survival and progression. The most established strategy is secreting vascular endothelial growth factor (VEGF), which stimulates angiogenesis to the TME. In a study on lung cancer cells, it has been elucidated that this process is HIF-dependent through direct binding of HIF-1 $\alpha$ to the VEGF gene promoter ${ }^{25}$. As noted before, HIF-1 $\alpha$ requires mitochondria for proper action ${ }^{9}$.

Collectively, this section demonstrated that functional mitochondria are vital for cancer cells to survive in a harsh TME.

\subsection{Immune Evasion}

Functional mitochondria support cancer cells to evade immune surveillance in the following ways:

2.2.1. TME acidification: In low-pH TME, immune cells lose their function and enter a state of anergy followed by apoptosis. Cancer cells with functional mitochondria have increased glycolytic flux, which leads to TME acidosis through lactate efflux (the end product of aerobic glycolysis) to the extracellular milieu ${ }^{8,26}$. Furthermore, functional mitochondria can promote TME acidosis by increasing lactate production through HIF-1 $\alpha$ mediated lactate dehydrogenase (LDH) activation and increasing lactate efflux through CA IX mediated MCT activation. ${ }^{8,19}$. As noted earlier, functional mitochondria are essential for proper HIF-1 $\alpha$ and CA IX activity ${ }^{9}$.

2.2.2. Glucose influx: In the metabolic competition with immune cells, cancer cells overexpress the glucose transporters (such as GLUT-1) to support their metabolism and make glucose out of the reach of immune cells. Given the importance of glucose for energy production required for proper immune cells function, glucose depletion leads to immune 
dysfunction ${ }^{27}$. A study on ovarian cancer cells revealed that HIF- $1 \alpha$ is the regulating factor of GLUT-1 expression ${ }^{28}$. We noted before that mitochondria support HIF-1 $\alpha$ expression and function in cancer cells ${ }^{9}$.

2.2.3. Mitochondrial hijacking: Ample evidence has revealed that $\mathrm{T}$ cells (as the lead of antitumor immunity) require energy for the proper activation against cancer cells 29 . Mitochondrial hijacking from $\mathrm{T}$ cells suppresses immune surveillance by depleting the immune cells' energy sources. In addition, mitochondrial hijacking from T cells can further block their antitumor function by overexpressing programmed cell death protein-1 (PD-1) on T cells ${ }^{30}$. Mitochondrial trafficking through nanotubes is a GTP-dependent process, and GTP molecules are mainly produced in the mitochondrial Krebs cycle ${ }^{2,24}$.

2.2.4. Recruitment of myeloid-derived suppressor cells (MDSC) toward TME: MDSCs are one of the principal members of TME. They support tumorigenesis by (1) inhibiting T cells via PD-L1 expression, uptaking essential amino acids (e.g., cysteine, L-arginine, and tryptophan), and excreting immunosuppressants (e.g., IL-10, TGF- $\beta$, nitric oxide); (2) inhibiting natural killer (NK) cells via TGF- $\beta$ excretion, and (3) dendritic cells via IL-10 and nitric oxide excretion. Tumor-infiltrating MDSCs also recruit regulatory $\mathrm{T}$ cells (Tregs) by releasing CC chemokine receptor 5 (CCR5) ligands. Tregs also have immunoinhibitory effects ${ }^{31}$. Cancer cells lead to MDSCs recruitment into TME by releasing chemokines. A study on hepatocellular carcinoma demonstrated that releasing chemokines by cancer cells is regulated by HIF- $1 \alpha{ }^{32}$. Earlier, we mentioned that HIF- $1 \alpha$ requires mitochondria's support for the proper action ${ }^{9}$.

2.2.5. Expression of immune checkpoints: Recent evidence has put forward mitochondria participation in expression of programmed cell death protein-ligand 1 (PD-L1) on cancer cells. In a study on a melanoma mouse model, investigators demonstrated that mitochondrial DNA (mtDNA) can be released into the cytosol and triggers PD-L1 expression through the STING-IFN pathway. MtDNA releasing into the cytosol is ATP-dependent, which elucidate the importance of mitochondria in PD-L1 expression on cancer cells ${ }^{33}$. Besides, it has been indicated that PD-L1 expression on MDSC is HIF-dependent ${ }^{34}$. MDSCs' mitochondria can participate in PD-L1 expression by securing HIF- $1 \alpha$ function by producing mtROS ${ }^{35}$. In a colon cancer mouse model, VEGF-A leads to PD-1 expression on tumorinfiltrating $\mathrm{CD}^{+} \mathrm{T}$ cells ${ }^{36}$. One might link this phenomenon to the cancer cells' mitochondria; As mentioned above, VEGF-A expression is HIF-dependent, mainly controlled by mitochondria ${ }^{5,9}$.

2.2.6. Defective antigen presentation: One of the main mechanisms cancer cells evade the immune system is losing major histocompatibility class I (MHC-I) molecules. In a fibrosarcoma mouse model, It has been reported that hypoxia downregulates MHC-1 through HIF- $1 \alpha{ }^{37}$. As mentioned earlier, mitochondria are essential for proper HIF-1 $\alpha$ action ${ }^{9}$. 
2.2.7. Immunosuppressive mediators: Besides MDSCs and Tregs, cancer cells per se can suppress immune control by releasing immunosuppressants. It has been shown that HIF$1 \alpha$ increases gene expression of IL- 10 and TGF- $\beta$ by direct binding to their promoter ${ }^{5}$. As mentioned, HIF- $1 \alpha$ expression and function is dependent on functional mitochondria ${ }^{9}$.

Collectively, this section demonstrated that functional mitochondria are crucial for cancer immune evasion.

\subsection{Cancer Progression}

Mitochondria generate $90 \%$ of the total cellular ROS volume, mainly by complexes I and III of the mitochondrial respiratory chain ${ }^{38,39}$. ROSs are a group of oxygen-containing, highlyactive, short-lived molecules. ROS in cancer cells is a double-edged sword. On the one hand, it helps cancer progression in moderate levels; on the other hand, it leads to cancer cell apoptosis at high levels ${ }^{39}$. Functional mitochondria give rise to elevated 'ROS balance'. It means they elevate and maintain ROS concentration at moderate levels to help cancer progression but impede damage to the cancer cells' component ${ }^{40}$. This section explains how mitochondrial ROSs (mtROSs) improve cancer progression. The former gives rise to (1) genomic instability, (2) cell cycle checkpoint evasion, and (3) and mediates epithelialto-mesenchymal transition that is a prelude for metastasis.

2.3.1. Genomic instability: It is a hallmark of cancer. Mitochondria can assist genomic instability in several ways. First, elevated mtROS directly damage mitochondrial and nuclear DNA by oxidizing nucleosides ${ }^{41}$. Another mechanism by which mitochondria lead to DNA mutation is by inducing 'minority mitochondrial outer membrane permeabilization (MOMP)'. Compared to MOMP (which is the trigger point of apoptosis), minority MOMP causes DNA mutation without apoptosis ${ }^{42}$. In esophageal cancer cells, an increase in ROS production and Mcl-1 expression are associated with minority MOMP ${ }^{43}$. Functional mitochondria are involved in minority MOMP through elevating ROS production and securing HIF- $1 \alpha$ function, which directly increases Mcl-1 expression ${ }^{44}$. Besides genetic mutations, the inactivation of DNA damage repair pathways is essential to establish the genomic instability in cancer cells. The direct effect of mitochondria on DNA damage repair has not been elucidated. Interestingly, one might assume this effect by considering the following two assumptions: (1) HIF-1 $\alpha$ leads to downregulation of mismatch repair (MMR) genes ${ }^{45}$; (2) mitochondria secure HIF-1 $\alpha$ function.

2.3.2. Quiescence evasion: In a growth permissive TME, cancer cells exit the quiescence state and restart the cell cycle to proliferate. Mitochondria can participate in quiescence evasion in two ways: (1) extrinsic pathway: $\beta 1$ integrin is a cell surface receptor that interacts with TME and mediates cancer cells invasion and metastasis ${ }^{46}$. In growth permissive TME, $\beta 1$ integrin activates the FAK-Src-MAPK pathway, prompting cancer cells to restart the cell cycle ${ }^{47}$. An in vivo study on osteosarcoma cells demonstrated that blocking OXPHOS resulted in $\beta 1$ integrin overexpression ${ }^{48}$. This process is similar to aerobic glycolysis, in which HIF-1 $\alpha$ shifts cancer cells' metabolism from OXPHOS to glycolysis. Therefore, one may 
conclude the cross-talk between HIF- $1 \alpha$ and $\beta 1$ integrin. As noted before, functional mitochondria are essential for HIF- $1 \alpha$ expression, stability, and function ${ }^{9}$. (2) Intrinsic pathway: elevated ROS level can lead to cell cycle reactivation. Functional mitochondria can contribute to quiescence evasion by producing more ROS ${ }^{49}$.

2.3.3. Metastasis: Epithelial-to-mesenchymal transition (EMT) is a transforming process that augments cancer cells' cell-cell adhesion loss, local migration, vascular invasion, and resistance to apoptotic stimuli. Therefore, EMT provides the characteristics essential for metastasis ${ }^{50}$. It has been established that ROS promotes EMT through mitogen-activated protein kinases (MAPK) and PI3K-Akt-mTOR activation, which in turn activates downstream SNAIL, matrix metalloproteinase 2 (MMP2), and MMP9 enzymes initiating EMT ${ }^{39}$. Besides, in breast cancer cells, ROS can lead to EMT through the VEGFA-SOX2SNAI2 pathway ${ }^{51}$. As noted, functional mitochondria elevate the intracellular ROS balance and maintain it at a moderate level ${ }^{40}$. Another mitochondria-mediated mechanism has been demonstrated in cancer metastasis. In an invasive breast cancer model, the cross-link between $\beta 1$ integrin and extracellular matrix was involved in cancer proliferation, invasion, and metastasis ${ }^{52}$. This process is mediated by lysyl oxidase (LOX), which per se is upregulated by HIF- $1 \alpha^{53}$. Mitochondria enhance LOX function by securing HIF-1 $\alpha$ function 9 .

Collectively, this section demonstrated how functional mitochondria assist cancer progression.

\subsection{Resistance to Treatment}

2.4.1. Chemotherapy: Mitochondria protect cancer cells from chemotherapy in several ways: (1) Most chemotherapy medicaments trigger cell death through oxidative stress. This is mediated by damage to cancer cell components and promoting apoptosis ${ }^{54}$. As noted in section 2.1.2, mitochondria are involved in enhanced redox homeostasis of cancer cells by direct expression of antioxidant enzymes and glutathione, providing nicotinamide adenine dinucleotide phosphate (NADPH) to preserve glutathione at a reduced state, and increasing pyruvate production through glycolysis flux $6,7,9,12$; (2) multidrug resistance (MDR) is mainly due to ATP-dependent multidrug efflux pumps that pump out chemotherapy agents. In a small cell lung cancer model, MDR efflux pumps were upregulated through the NRF2 pathway ${ }^{55}$. As noted before, functional mitochondria stimulate NRF2 function by increasing mtROS ${ }^{22}$; (3) functional mitochondria assist MDR by providing sufficient ATP for ATPdependent efflux pumps ${ }^{56}$; and (4) in breast cancer cells, mitochondria led to doxorubicin resistance by inducing cell cycle arrest ${ }^{57}$.

2.4.2. Radiotherapy: Ionizing radiation can damage cancer cells by direct damage to DNA or dominantly through ROS generation and indirect damages to cellular and mitochondrial components ${ }^{58}$. As mentioned in section 2.1.2, mitochondria protect cancer cells from radiotherapy by scavenging the generated ROS 6,7,9,12. 
2.4.3. Immunotherapy: In addition to radiotherapy and chemotherapy, mitochondria can enhance the resistance to immunotherapy. This notion was demonstrated in an in vivo experiment in which blocking the mitochondria trafficking from $\mathrm{T}$ cells to cancer cells improved the efficacy of anti-PD-1 immunotherapy ${ }^{2}$. As noted in section 2.2.3, functional mitochondria can potentially take part in the mitochondrial hijacking process by providing sufficient GTP for Miro1 ${ }^{24}$. This process depletes T cells' energy and impedes long-term immune surveillance ${ }^{30}$. This defensive mechanism of cancer can also involve other modes of immunotherapy, including adoptive cell therapy and cancer vaccines.

Collectively, this section demonstrated how functional mitochondria improve cancer resistance to treatments.

\section{Discussion}

\subsection{An Energy Battle Between Immune and Cancer Cells}

In this article, we demonstrated the crucial role of mitochondria in cancer cells' survival, progression, and confrontation with immune cells. In the struggle between immune and cancer cells, each party with a higher energy level can win the battle. More functional mitochondria empower the cancer cells and enable them to overcome their opponent, the immune cells. As alluded to above, mitochondrial hijacking from immune cells upgrades the cancer cells' resistance to anti-PD-1 antibodies ${ }^{2}$. This finding supports the hypothesis that $\mathrm{T}$ cells' mitochondria content determines response to anti-PD-1 immunotherapy. In Jan 2021, Akbari and Taghizadeh-Hesary et al. demonstrated that T cells' mitochondrial activation can improve the response to anti-PD-1 antibodies by improving recognition (through PD-1 downregulation on $\mathrm{T}$ cells) and providing energy for long-term $\mathrm{T}$ cell activation ${ }^{30}$. This strategic finding can introduce a new, potential theory in oncology, the 'energy battle'. In this theory, shifting the energy balance toward the immune cells can improve clinical outcomes. Theoretically, leveling up the immune cells (against cancer cells) can potentially serve as monotherapy. Immune cells with stronger mitochondria are more efficient in all phases of cancer cell recognition (through PD-1 downregulation), activation, proliferation, migration, and cancer cell killing ${ }^{30,59,60}$. All these phases are ATP-dependent 29,30 . On the other hand, cancer cells with weaker mitochondria cannot tolerate the bulk of ROSs generated in the hypoxic TME and procced to apoptosis.

Shifting the energy balance toward the immune cells is accessible by improving T cells' mitochondria in quantity and quality. For the primer, the T cells' mitochondria numbers can be saved by blocking mitochondrial hijacking ${ }^{2}$. The mitochondria quality can increase by two strategies; (1) improving the lifestyle by regular exercise ${ }^{61}$, low-SDA (specific dynamic action) diet ${ }^{62}$, good sleep ${ }^{63}$, healthy weight ${ }^{64}$, and smoking cessation ${ }^{65}$; and (2) mitochondria boosting agents [e.g., activators of adenosine monophosphate-activated 
protein kinase (AMPK), mammalian target of rapamycin (mTOR), and peroxisome proliferator-activated receptor-gamma coactivator 1-alpha (PGC-1 $\alpha){ }^{66}$.

\subsection{Resistance to the Current Cancer Treatment}

Despite considerable advances in cancer treatment, cancer recurrence is frequently seen. It has been reported in $50 \%$ of patients with soft tissue sarcoma, $85 \%$ of patients with ovarian cancer, and almost all patients with glioblastoma ${ }^{67}$. Cancer cells can develop resistance to the available treatments through specific genetic and epigenetic changes. For instance, resistance to radiotherapy by amplifying ROS clearing system, resistance to chemotherapy by MDR efflux pumps, cell cycle arrest, and ROS clearing, and resistance to immunotherapy by depleting T cells' mitochondrial content through mitochondrial hijacking. This article demonstrated that mitochondria are common actors in these resistance mechanisms. Besides, in response to targeted therapies, cancer cells can circumvent the blocked pathway through many different mechanisms ${ }^{68}$, including (1) restoration of the targeted molecules (e.g., BCR-ABL kinase reactivation in imatinib therapy of chronic myelogenous leukemia) ${ }^{69}$, (2) activation of upstream and downstream signaling proteins (e.g., MAP kinase signaling restoration in vemurafenib therapy of melanoma) ${ }^{70}$, (3) histologic transformation (e.g., transformation into small cell carcinoma in tyrosine kinase therapy of EGFR mutant NSCLC) 71, and (4) adaptive signaling to promote survival (e.g., HIF-dependent cell cycle arrest in doxorubicin therapy of breast cancer) ${ }^{57}$. Current literature indicates that targeting cancer through different mechanisms can improve clinical outcomes. To better delineate this notion, the following example is presented. Over the last two decades, the six months-PFS (progression-free survival) of patients with platinum-resistant ovarian cancer has improved from $30 \%$ in chemotherapy-alone ${ }^{72}$, to $47 \%$ in the chemotherapy plus anti-VEGF 73 , to $53 \%$ in the chemotherapy plus anti-VEGF plus anti-PD-1 ${ }^{74}$. This improvement in oncological outcome is at the expense of more toxicities.

\subsection{Future Directions}

Thanks to the current understanding of mitochondria's role in cancer metabolism, antimitochondrial therapy can be a potential therapeutic approach in oncology. It can serve as an adjuvant to radiotherapy by preventing ROS clearing, adjuvant to chemotherapy by inactivating cell cycle arrest, efflux pump, and ROS clearing, and adjuvant to immunotherapy by preventing mitochondria hijacking (refer to section 2.4.3.). Anti-mitochondria therapy has the potential to serve as a definitive therapy as well. This can be mediated by inhibiting the pathways that are the cornerstone of cancer cell metabolism to live and develop. By completely inhibiting mitochondrial function, at least twenty-two vital mechanisms become synchronously affected (Figure 1), possibly without circumventing pathways for cancer. In this condition, the cancer cell cannot survive in the hypoxic, acidic TME, cannot evade the immune system, cannot improve its malignancy. Therefore, anti-mitochondrial therapy can revolutionize future cancer treatment.

Accumulating evidence indicates that cancer cells can maintain the mitochondria ultrastructure and function in hypoxic conditions ${ }^{6}$. In addition, cancer cells can provide 
more functional mitochondria for themselves by hijacking from normal cells ${ }^{2}$. By identifying and blocking the mitochondria-boosting pathways, humans can overcome cancer in the future.

\section{Conclusions}

Herein, we highlighted the importance of mitochondrion in cancer cell metabolism. It provides crucial benefits for cancer cells in terms of survival in hypoxic TME, immune evasion, progression, and resistance to treatment. We noted that cancer cells can maintain their mitochondrial function under hypoxia and even hijack functional mitochondria from normal cells. With these in mind, mitochondria are necessary for cancer cells to survive. Given its multifaceted role in cancer cells, mitochondria are possibly cancer's Achilles' heel. Practitioners can overcome cancer by identifying and blocking the strategies by which cancer cells maintain their mitochondria's quality and quantity. Further studies are warranted to examine this theory.

Declarations

Funding: None

Conflicts of interest: The authors declare that they have no competing interests.

Availability of data and material: Not applicable

CRediT author statement:

Conceptualization: F.TH, M.B, and H.A

Methodology: F.TH

Software: N/A

Validation: F.TH

Formal analysis: N/A

Investigation: F.TH

Resources: F.TH, M.B

Data Curation: N/A

Writing-original draft: F.TH

Writing-review \& editing: F.TH

Visualization: F.TH

Supervision: M.B

Project administration: F.TH

Funding acquisition: N/A

Acknowledgement: None 


\section{References}

1. Halvorson HM, Wyatt KH, Kuehn KA. Ecological significance of autotroph-heterotroph microbial interactions in freshwaters. Freshwater Biology. 2020;65(7):1183-1188

2. Saha T, Dash C, Jayabalan R, et al. Intercellular nanotubes mediate mitochondrial trafficking between cancer and immune cells. Nature nanotechnology. 2021:1-9

3. Wu Y-T, Wu S-B, Wei Y-H. Metabolic reprogramming of human cells in response to oxidative stress: implications in the pathophysiology and therapy of mitochondrial diseases. Current pharmaceutical design. 2014;20(35):5510-5526

4. Akbari $\mathrm{H}$, Taghizadeh-Hesary F, Heike $\mathrm{Y}$, Bahadori M. Cell energy: A new hypothesis in decoding cancer evolution. Archives of Iranian Medicine (AIM). 2019;22(12)

5. You L, Wu W, Wang X, et al. The role of hypoxia-inducible factor 1 in tumor immune evasion. Medicinal research reviews. 2021;41(3):1622-1643

6. Li P, Zhang D, Shen $L$, et al. Redox homeostasis protects mitochondria through accelerating ROS conversion to enhance hypoxia resistance in cancer cells. Scientific reports. 2016;6(1):1-13

7. Lee S, Hallis SP, Jung KA, Ryu D, Kwak MK. Impairment of HIF-1 a -mediated metabolic adaption by NRF2-silencing in breast cancer cells. Redox Biol.

2019;24:101210.10.1016/j.redox.2019.101210

8. Ishida T, Nakao S, Ueyama T, Harada Y, Kawamura T. Metabolic remodeling during somatic cell reprogramming to induced pluripotent stem cells: involvement of hypoxia-inducible factor 1. Inflammation and Regeneration. 2020;40(1):1-8

9. van Gisbergen MW, Offermans K, Voets AM, et al. Mitochondrial Dysfunction Inhibits HypoxiaInduced HIF-1 a Stabilization and Expression of Its Downstream Targets. Frontiers in Oncology. 2020;10.10.3389/fonc.2020.00770

10. Vaupel P, Multhoff G. Revisiting the Warburg effect: historical dogma versus current understanding. The Journal of Physiology. 2021;599(6):1745-1757

11. Paredes F, Williams HC, San Martin A. Metabolic adaptation in hypoxia and cancer. Cancer letters. 2021

12. Mitsuishi $\mathrm{Y}$, Taguchi K, Kawatani $\mathrm{Y}$, et al. Nrf2 redirects glucose and glutamine into anabolic pathways in metabolic reprogramming. Cancer cell. 2012;22(1):66-79

13. Wang X, Perez E, Liu R, Yan L, Mallet RT, Yang SH. Pyruvate protects mitochondria from oxidative stress in human neuroblastoma SK-N-SH cells. Brain Res. 2007;1132(1):1-

9.10.1016/j.brainres.2006.11.032

14. Tauffenberger A, Fiumelli H, Almustafa S, Magistretti PJ. Lactate and pyruvate promote oxidative stress resistance through hormetic ROS signaling. Cell Death \& Disease. 2019;10(9):653.10.1038/s41419-019-1877-6

15. Ramos-Ibeas P, Barandalla M, Colleoni S, Lazzari G. Pyruvate antioxidant roles in human fibroblasts and embryonic stem cells. Mol Cell Biochem. 2017;429(1-2):137150.10.1007/s11010-017-2942-z

16. Druker J, Wilson JW, Child F, Shakir D, Fasanya T, Rocha S. Role of Hypoxia in the Control of the Cell Cycle. International Journal of Molecular Sciences. 2021;22(9):4874

17. Koshiji M, Kageyama Y, Pete EA, Horikawa I, Barrett JC, Huang LE. HIF-1 a induces cell cycle arrest by functionally counteracting Myc. The EMBO journal. 2004;23(9):1949-1956

18. Carcereri de Prati A, Butturini E, Rigo A, et al. Metastatic breast cancer cells enter into dormant state and express cancer stem cells phenotype under chronic hypoxia. Journal of cellular biochemistry. 2017;118(10):3237-3248 
19. Becker HM. Carbonic anhydrase IX and acid transport in cancer. British Journal of Cancer. 2020;122(2):157-167.10.1038/s41416-019-0642-z

20. Ding WX, Ni HM, Li M, et al. Nix is critical to two distinct phases of mitophagy, reactive oxygen species-mediated autophagy induction and Parkin-ubiquitin-p62-mediated mitochondrial priming. J Biol Chem. 2010;285(36):27879-27890.10.1074/jbc.M110.119537

21. Towers CG, Fitzwalter BE, Regan D, et al. Cancer Cells Upregulate NRF2 Signaling to Adapt to Autophagy Inhibition. Dev Cell. 2019;50(6):690-703.e696.10.1016/j.devcel.2019.07.010

22. Kasai S, Shimizu S, Tatara Y, Mimura J, Itoh K. Regulation of Nrf2 by Mitochondrial Reactive Oxygen Species in Physiology and Pathology. Biomolecules. 2020;10(2):320

23. Nazio F, Bordi M, Cianfanelli V, Locatelli F, Cecconi F. Autophagy and cancer stem cells: molecular mechanisms and therapeutic applications. Cell Death \& Differentiation. 2019;26(4):690-702

24. Lambeth DO. What is the function of GTP produced in the Krebs citric acid cycle? IUBMB Life. 2002;54(3):143-144.10.1080/15216540214539

25. Zhu H, Zhang S. Hypoxia inducible factor-1 a /vascular endothelial growth factor signaling activation correlates with response to radiotherapy and its inhibition reduces hypoxia-induced angiogenesis in lung cancer. J Cell Biochem. 2018;119(9):7707-7718.10.1002/jcb.27120

26. Huber V, Camisaschi C, Berzi A, et al. Cancer acidity: An ultimate frontier of tumor immune escape and a novel target of immunomodulation. Paper presented at: Seminars in cancer biology2017.

27. Klein $\mathrm{K}, \mathrm{He} \mathrm{K}$, Younes Al, et al. Role of mitochondria in cancer immune evasion and potential therapeutic approaches. Frontiers in immunology. 2020:2622

28. Yu X-J, Song J-C, Du J, Shi Y-Q, Liu Y-X, Shen Y. GLUT-1 and its regulating factor HIF-1 a expression in epithelial ovarian tumors: GLUT-1 is associated with molecular typing and grade of epithelial ovarian cancer. Int J Clin Exp Pathol [Internet]. 2017;10(4):4479-4487

29. Herbel C, Patsoukis N, Bardhan K, Seth P, Weaver JD, Boussiotis VA. Clinical significance of T cell metabolic reprogramming in cancer. Clinical and translational medicine. 2016;5(1):1-23

30. Akbari H, Taghizadeh-Hesary F, Bahadori M. Mitochondria determine response to antiprogrammed cell death protein-1 (anti-PD-1) immunotherapy: An evidence-based hypothesis. Mitochondrion. 2022;62:151-158

31. Yang Y, Li C, Liu T, Dai X, Bazhin AV. Myeloid-Derived Suppressor Cells in Tumors: From Mechanisms to Antigen Specificity and Microenvironmental Regulation. Frontiers in Immunology. 2020;11.10.3389/fimmu.2020.01371

32. Chiu DKC, Xu IMJ, Lai RKH, et al. Hypoxia induces myeloid-derived suppressor cell recruitment to hepatocellular carcinoma through chemokine (C-C motif) ligand 26. Hepatology. 2016;64(3):797813

33. Cheng AN, Cheng L-C, Kuo C-L, et al. Mitochondrial Lon-induced mtDNA leakage contributes to PD-L1-mediated immunoescape via STING-IFN signaling and extracellular vesicles. Journal for immunotherapy of cancer. 2020;8(2)

34. Noman MZ, Desantis G, Janji B, et al. PD-L1 is a novel direct target of HIF-1 a, and its blockade under hypoxia enhanced MDSC-mediated T cell activation. J Exp Med. 2014;211(5):781790.10.1084/jem.20131916

35. Ohl K, Tenbrock K. Reactive oxygen species as regulators of MDSC-mediated immune suppression. Frontiers in immunology. 2018:2499

36. Voron T, Colussi O, Marcheteau E, et al. VEGF-A modulates expression of inhibitory checkpoints on CD8+ T cells in tumors. Journal of Experimental Medicine. 2015;212(2):139-148 
37. Sethumadhavan S, Silva M, Philbrook P, et al. Hypoxia and hypoxia-inducible factor (HIF) downregulate antigen-presenting $\mathrm{MHC}$ class I molecules limiting tumor cell recognition by $\mathrm{T}$ cells. PLoS One. 2017;12(11):e0187314

38. Wang $\mathrm{H}$, Jiang $\mathrm{H}$, Van De Gucht $\mathrm{M}$, De Ridder M. Hypoxic radioresistance: can ROS be the key to overcome it? Cancers. 2019;11(1):112

39. Aggarwal V, Tuli HS, Varol A, et al. Role of reactive oxygen species in cancer progression: molecular mechanisms and recent advancements. Biomolecules. 2019;9(11):735

40. Dunn JD, Alvarez LA, Zhang X, Soldati T. Reactive oxygen species and mitochondria: A nexus of cellular homeostasis. Redox biology. 2015;6:472-485

41. Bonora M, Missiroli S, Perrone M, Fiorica F, Pinton P, Giorgi C. Mitochondrial control of genomic instability in cancer. Cancers. 2021;13(8):1914

42. Kalkavan H, Green DR. MOMP, cell suicide as a BCL-2 family business. Cell Death \& Differentiation. 2018;25(1):46-55.10.1038/cdd.2017.179

43. Xu Y, Surman DR, Diggs L, et al. Bile acid-induced "Minority MOMP" promotes esophageal carcinogenesis while maintaining apoptotic resistance via Mcl-1. Oncogene. 2020;39(4):877890.10.1038/s41388-019-1029-6

44. Wu F, Tong DD, Ni L, Wang LM, Wang MC. HIF-1 a suppresses myeloma progression by targeting Mcl-1. Int J Clin Exp Pathol. 2020;13(7):1483-1491

45. Koshiji M, To KK-W, Hammer S, et al. HIF-1 a induces genetic instability by transcriptionally downregulating MutS a expression. Molecular cell. 2005;17(6):793-803

46. Ganguly KK, Pal S, Moulik S, Chatterjee A. Integrins and metastasis. Cell adhesion \& migration. 2013;7(3):251-261

47. Fiore APZP, Ribeiro PdF, Bruni-Cardoso A. Sleeping Beauty and the Microenvironment Enchantment: Microenvironmental Regulation of the Proliferation-Quiescence Decision in Normal Tissues and in Cancer Development. Frontiers in Cell and Developmental Biology. 2018;6.10.3389/fcell.2018.00059

48. Nunes JB, Peixoto J, Soares $\mathrm{P}$, et al. OXPHOS dysfunction regulates integrin- $\beta 1$ modifications and enhances cell motility and migration. Human Molecular Genetics. 2014;24(7):19771990.10.1093/hmg/ddu612

49. Zhou D, Shao L, Spitz DR. Reactive oxygen species in normal and tumor stem cells. Adv Cancer Res. 2014;122:1-67.10.1016/b978-0-12-420117-0.00001-3

50. Mittal V. Epithelial mesenchymal transition in tumor metastasis. Annual Review of Pathology: Mechanisms of Disease. 2018;13:395-412

51. Kim M, Jang K, Miller $P$, et al. VEGFA links self-renewal and metastasis by inducing Sox 2 to repress miR-452, driving Slug. Oncogene. 2017;36(36):5199-5211.10.1038/onc.2017.4

52. Erler JT, Bennewith KL, Nicolau M, et al. Lysyl oxidase is essential for hypoxia-induced metastasis. Nature. 2006;440(7088):1222-1226

53. Amendola PG, Reuten R, Erler JT. Interplay Between LOX Enzymes and Integrins in the Tumor Microenvironment. Cancers (Basel). 2019;11(5).10.3390/cancers11050729

54. Yang $\mathrm{H}$, Villani $\mathrm{RM}$, Wang $\mathrm{H}$, et al. The role of cellular reactive oxygen species in cancer chemotherapy. Journal of Experimental \& Clinical Cancer Research. 2018;37(1):1-10

55. Ji L, Li H, Gao P, et al. Nrf2 pathway regulates multidrug-resistance-associated protein 1 in small cell lung cancer. PLoS One. 2013;8(5):e63404.10.1371/journal.pone.0063404

56. Perillo B, Di Donato M, Pezone A, et al. ROS in cancer therapy: The bright side of the moon. Experimental \& Molecular Medicine. 2020;52(2):192-203

57. Dornfeld K, Bjork J, Folkert G, Skildum A, Wallace KB. Mitochondrial activities play a pivotal role in regulating cell cycle in response to doxorubicin. Cell Cycle. 2021;20(11):1067-1079 
58. Desouky O, Ding N, Zhou G. Targeted and non-targeted effects of ionizing radiation. Journal of Radiation Research and Applied Sciences. 2015;8(2):247-254

59. Desdín-Micó G, Soto-Heredero G, Mittelbrunn M. Mitochondrial activity in T cells. Mitochondrion. 2018;41:51-57

60. Surace L, Doisne J-M, Escoll P, et al. Polarized mitochondria as guardians of NK cell fitness. Blood advances. 2021;5(1):26-38

61. Memme JM, Erlich AT, Phukan G, Hood DA. Exercise and mitochondrial health. The Journal of physiology. 2021;599(3):803-817

62. Luoma RL, Butler MW, Stahlschmidt ZR. Plasticity of immunity in response to eating. Journal of Experimental Biology. 2016;219(13):1965-1968

63. Rodrigues NR, Macedo GE, Martins IK, et al. Short-term sleep deprivation with exposure to nocturnal light alters mitochondrial bioenergetics in Drosophila. Free Radical Biology and Medicine. 2018;120:395-406

64. de Mello AH, Costa AB, Engel JDG, Rezin GT. Mitochondrial dysfunction in obesity. Life sciences. 2018;192:26-32

65. Malińska D, Więckowski MR, Michalska B, et al. Mitochondria as a possible target for nicotine action. Journal of bioenergetics and biomembranes. 2019;51(4):259-276

66. Chamoto K, Chowdhury PS, Kumar A, et al. Mitochondrial activation chemicals synergize with surface receptor PD-1 blockade for T cell-dependent antitumor activity. Proceedings of the National Academy of Sciences. 2017;114(5):E761-E770

67. van der Merwe M, van Niekerk G, Fourie C, du Plessis M, Engelbrecht A-M. The impact of mitochondria on cancer treatment resistance. Cellular Oncology. 2021;44(5):983-995

68. Sabnis AJ, Bivona TG. Principles of Resistance to Targeted Cancer Therapy: Lessons from Basic and Translational Cancer Biology. Trends Mol Med. 2019;25(3):185-

197.10.1016/j.molmed.2018.12.009

69. Shah NP, Tran C, Lee FY, Chen P, Norris D, Sawyers CL. Overriding imatinib resistance with a novel ABL kinase inhibitor. Science. 2004;305(5682):399-401

70. Nazarian R, Shi H, Wang Q, et al. Melanomas acquire resistance to B-RAF (V600E) inhibition by RTK or N-RAS upregulation. Nature. 2010;468(7326):973-977

71. Sequist LV, Waltman BA, Dias-Santagata D, et al. Genotypic and histological evolution of lung cancers acquiring resistance to EGFR inhibitors. Sci Transl Med.

2011;3(75):75ra26.10.1126/scitransImed.3002003

72. Mutch DG, Orlando M, Goss T, et al. Randomized phase III trial of gemcitabine compared with pegylated liposomal doxorubicin in patients with platinum-resistant ovarian cancer. J Clin Oncol. 2007;25(19):2811-2818.10.1200/jco.2006.09.6735

73. Kudoh $\mathrm{K}$, Takano $\mathrm{M}$, Kouta $\mathrm{H}$, et al. Effects of bevacizumab and pegylated liposomal doxorubicin for the patients with recurrent or refractory ovarian cancers. Gynecol Oncol. 2011;122(2):233237.10.1016/j.ygyno.2011.04.046

74. Michels J, Ghiringhelli F, Frenel J-S, et al. Pembrolizumab in combination with bevacizumab and pegylated liposomal doxorubicin in patients with platinum-resistant epithelial ovarian cancer. Journal of Clinical Oncology. 2021;39(15_suppl):5522-5522.10.1200/JCO.2021.39.15_suppl.5522 